\title{
Investigation on Enhancing Flexural Performance of CFRP Strengthened Concrete Beams
}

\author{
[J.C.P.H. Gamage, M.R.E.F. Ariyachandra]
}

\begin{abstract}
Strengthening of reinforced concrete structures is inevitable to preserve structural integrity and protracted service life due to the effects of numerous elements contained within the natural or artificial environment surrounding it. Carbon Fibre Reinforced Polymer (CFRP) composites have been progressively more utilized for decades as a strengthening material due to its outstanding characteristics such as high strength to weight ratio, reduced weight, excellent resistance to corrosion and environmental degradation and ease of handling during construction which have distinguished it well apart from the other strengthening techniques. However, the ultimate load capacity of CFRP strengthened concrete composites is comparatively lower than CFRP material due to premature debonding which reported as the most frequently observed failure mode in CFRP/concrete composites. In the current study, alternative bonding techniques were investigated to achieve enhanced bond performance by delaying premature debonding failure. A total of 18 CFRP strengthened concrete beams with different bonding techniques were tested using three point bending test. The test results show convincing evidence on strength enhancements. Moreover, a numerical model was also developed to inspect the performance of existing CFRP anchors. Finally, a parametric study was carried out and a new type of anchor; "Tri-leg anchor" is introduced. This paper summarizes the overview of the test programme, finite element modeling, results and analysis.
\end{abstract}

Keywords-CFRP, debonding, end wraps, polyester mesh, Tri-leg anchor

\section{Introduction}

As reported in literature [1,2,3,4], CFRP/concrete composites exhibit fundamentally two types of failure criterions. In the first category, the full composite action of concrete and FRP is maintained until the failure occurs (i.e. FRP rupture and crushing of compressive concrete). Such failure modes are entitled as "classical failure modes". The composite action between concrete and FRP is lost prior to failure in the second category, (i.e. de-bonding of FRP) and also known as "premature failure modes." Separation of concrete cover, separation of FRP plate and interfacial bond failure can be recognized as such failure behaviours of CFRP/concrete composites.

J.C.P.H. Gamage (Senior Lecturer)

University of Moratuwa

Sri Lanka

M.R.E.F Ariyachandra (Postgraduate Student)

University of Moratuwa

Sri Lanka
Above all, end-debonding failure (interface failure between CFRP sheet/plate and concrete substrate at the ends of CFRP sheet/plate) is the most commonly reported failure mode $[5,6]$ which significantly declines the ultimate load carrying capacity of CFRP/concrete composites ranging from $60 \%$ to $80 \%$ [7] while upgrading the brittleness at failure.

To address this decisive phenomenon, a great deal of research studies has been carried out grasping the use of mechanical fasteners, FRP anchors, Near Surface Mount (NSM) reinforcement and End Wraps [6]. End Wraps have been recognized as a beneficial way out to delay premature debonding since it enhances the shear capacity of CFRP/concrete composites [8]. However, previous research studies emphasize the necessity of establishing appropriate design guidelines to predict the strength gains due to end wraps [9] which has led to restrict possible industrial applications. In the current study, alternative bonding techniques were adopted in order to achieve enhanced bond performance by delaying premature debonding failure. A total of 18 CFRP strengthened concrete beams with different bonding techniques were tested using three point bending test. A numerical model was developed using ANSYS to inspect the performance of existing CFRP anchors. A parametric study was carried out via numerical analysis and a new type of anchor; "Tri-leg anchor" is introduced.

\section{Material properties and specimen characterization}

A total of 18 concrete beams with $100 \mathrm{~mm} \times 150 \mathrm{~mm} \times 750$ $\mathrm{mm}$ dimensions (width $\times$ depth $\times$ length) were prepared using grade 30 concrete mix containing maximum aggregate size of $20 \mathrm{~mm}$ and water/cement ratio of 0.55 . The configuration of reinforcements of concrete test specimens is shown in Fig. 1. These specimens were immersed in water for maturation period of 28 days. A total of 10 concrete blocks of $150 \mathrm{~mm} \times$ $150 \mathrm{~mm} \times 150 \mathrm{~mm}$ which contains two from each batch of concrete were also prepared and cured under the same condition. The measured average compressive strength of concrete was $33.6 \mathrm{~N} / \mathrm{mm}^{2}$.

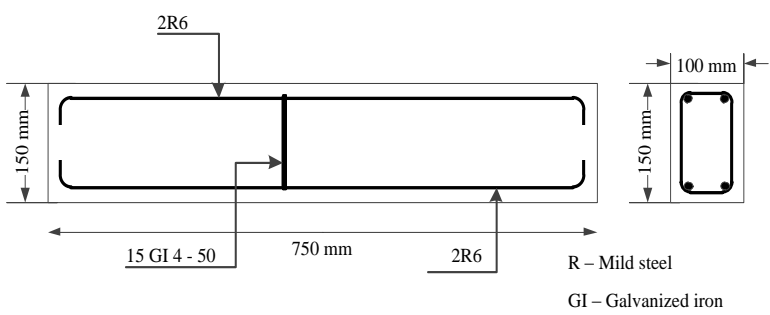

Figure 1. Configuration of reinforcements 
With the completion of curing process, concrete substrates of the beams were sand blasted for strengthening purpose. The properties of the CFRP sheet and two-part epoxy adhesive are listed in Table 1. Primer part A (hardener) and part B (base) were mixed to 1:2 ratio and a thin layer of primer was applied on the sand blasted concrete substrates and they were kept aside for 45 minutes. Saturant part A (hardener) and part B (base) were also mixed to 1:2 ratio. Then, primed concrete substrates and CFRP sheets were properly saturated with epoxy adhesive. Impregnated CFRP sheets were pressed on to the prepared concrete substrates and a ribbed roller was used to remove air entrapped in the bond line. Epoxy adhesive had a pot life of 40 minutes and the prepared CFRP/concrete test specimens were cured for 7 days at room temperature.

In this experimental investigation, alternative bonding techniques were adopted (as explained in section III) to achieve enhanced bond performance by delaying premature debonding failure. The FRP arrangement of CFRP/concrete control test specimens is shown in Fig. 2 and the test set up is illustrated in Fig. 3.

TABle 1. Material Properties Of CFRP SheEt AND Two Part EPOXY ADHESIVE [10]

\begin{tabular}{|l|c|c|c|}
\hline \multirow{2}{*}{\multicolumn{1}{|c|}{ Parameter }} & \multicolumn{3}{c|}{ Value } \\
\cline { 2 - 4 } & CFRP & Primer & Saturant \\
\hline Fiber Density & $2.1 \mathrm{~g} \mathrm{~cm}^{-3}$ & - & - \\
\hline Elastic Modulus & $640 \mathrm{GPa}$ & $595 \mathrm{MPa}$ & $3724 \mathrm{MPa}$ \\
\hline Thickness & $0.19 \mathrm{~mm}$ & - & - \\
\hline Ultimate Tensile Strength & $2600 \mathrm{MPa}$ & $24.1 \mathrm{MPa}$ & $138 \mathrm{MPa}$ \\
\hline Ultimate Tensile Elongation & $0.4 \%$ & $4 \%$ & $3.8 \%$ \\
\hline Glass Transition Temperature & - & $77^{\circ} \mathrm{C}$ & $71^{\circ} \mathrm{C}$ \\
\hline
\end{tabular}

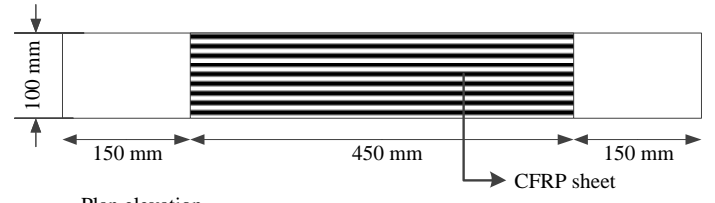
Plan elevation

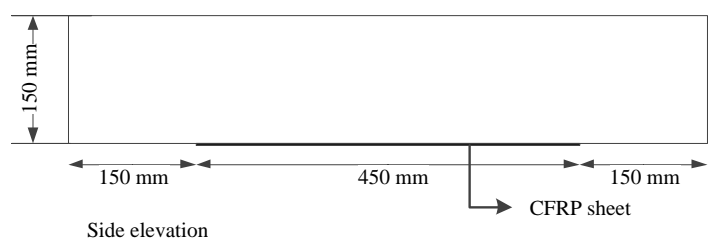

Figure 2. FRP arrangement of CFRP/concrete control test specimens

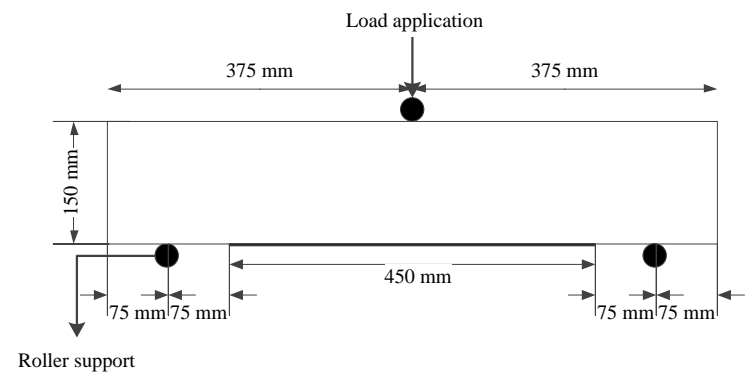

Figure3. Schematic diagram of three point bending test

\section{Test programme}

\section{A. Non-strengthened control specimens}

A total of six non-strengthened concrete test specimens were tested and the average failure loads at $0.3 \mathrm{~mm}$ crack width was measured. All control specimens were failed due to a single flexural crack which was initiated at the middle of the span as shown in Fig. 4. These six control samples were obtained from three different batches of concrete. The average failure loads observed from batch 1,2 and 3 were $14.71 \mathrm{kN}$, $12.36 \mathrm{kN}$ and $14.68 \mathrm{kN}$ respectively.

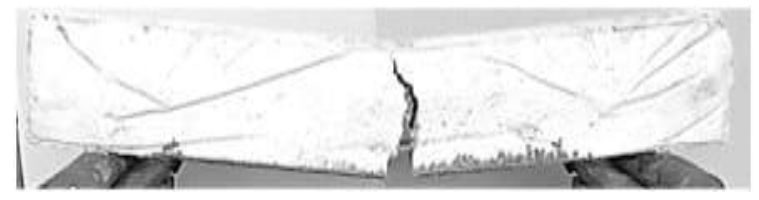

Figure 4. Flexural failure of non-strengthened control beam

\section{B. CFRP/concrete control specimens}

A total of six CFRP strengthened control concrete beams were tested. Cover separation failure was the typical mode of failure observed for these specimens as shown in Fig. 5. The reported average failure loads at $0.3 \mathrm{~mm}$ crack initiation were $40.22 \mathrm{kN}, 15.89 \mathrm{kN}$ and $31.40 \mathrm{kN}$ corresponding to concrete batches 1,2 and 3 respectively. The encountered failure mode can be recognized as a premature debonding failure $[1,2,3$, 4]. The overall strength gain was in the range of $60.68 \%$ to $213.46 \%$.

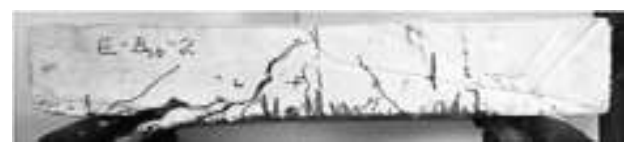

Figure 5.Cover separation failure of CFRP-strengthened control

\section{Use of vertical CFRP U wraps at ends}

The first alternative bonding technique was to provide two U shaped vertical CFRP end wraps of $150 \mathrm{~mm} \times 100 \mathrm{~mm}$ on top of longitudinal CFRP sheet in transverse direction as shown in Fig. 6. Two test specimens were tested which were cast using concrete from batch 1 and the average failure load was $46.11 \mathrm{kN}$. The reported failure mode was cover separation failure which originated from shear cracks followed near the supports as illustrated in Fig. 7. This is mainly due to the high stress concentration occurred at the CFRP plate ends. The key objective was to avoid end delamination which is the critical failure mode regarding CFRP/concrete composites. Even though the encountered failure pattern belongs to premature debonding failure category, the use of vertical CFRP U wraps has effectively delayed end delamination with a $14.64 \%$ strength increment in comparison of CFRP strengthened control specimens. This is about $213.46 \%$ strength gain after strengthening concrete beam with vertical $\mathrm{U}$ wraps.

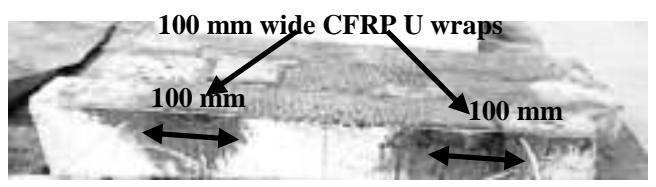

Figure 6. Vertical CFRP U wraps at ends 


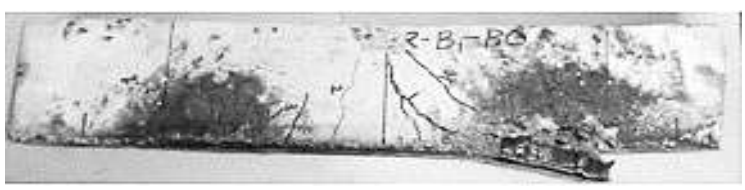

Figure 7. Cover separation failure of CFRP/concrete beam anchored with CFRP U wraps

\section{Use of a polyester mesh at bond line}

The second method of alternative bonding technique comprised a bond enhancer which facilitates a reinforcing medium for the bonding agent. Thus, a polyester mesh of 600 $\mathrm{mm} \times 60 \mathrm{~mm}$ was placed at the bond line i.e. between two part epoxy adhesive and CFRP sheet as illustrated in Fig. 8. A $1 \mathrm{~mm}$ thick polymer mesh was selected to be compatible with the existing polymeric materials. The principal aim was to provide a bond enhancing interface which can dissipate energy uniformly before failure. Two specimens which were prepared out of batch no. 2 concrete were tested under this category. The average failure load was $19.86 \mathrm{kN}$ owing $24.98 \%$ strength increment compared to CFRP strengthened control beams. This means an overall strength gain of $60.68 \%$ with concrete beam strengthened with CFRP sheet with polymer mesh. The observed flexural failure pattern consisted of mid span debonding (shown in Fig. 9) which propagated to the support ends. Thus, the defensive reason for the enddebonding and its successive strength gain can be directly attributed towards the presence of the polyester mesh which had effectively distributed the interfacial stresses well.

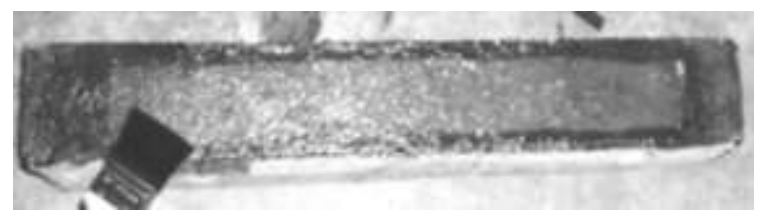

Figure $8.60 \mathrm{~mm}$ wide polyester mesh

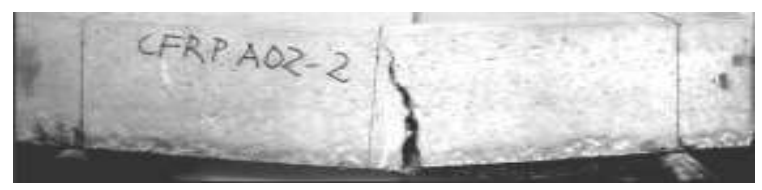

Figure 9. Flexural failure of CFRP/concrete beam with polyester mesh

\section{E. Use of inclined CFRP U wraps at ends}

The third implemented alternative bonding technique was to introduce $60 \mathrm{~mm}$ wide inclined CFRP U wraps at ends as shown in Fig. 10. The test specimens were prepared using concrete from batch 3 . The reported average failure load was $41.20 \mathrm{kN}$ and the observed failure mode comprised a flexural failure at mid span followed by CFRP rupture as illustrated in Fig. 11. This is mainly due to the enhanced bond strength at plate ends which had prevented the most crucial enddebonding failure mode commendably owing $31.23 \%$ strength increment compared to CFRP strengthened control beams. This is about $180.65 \%$ strength gain with respect to nonstrengthened concrete beam.

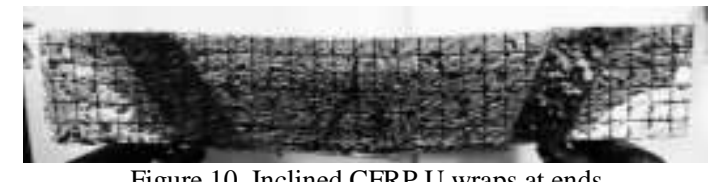

Figure 10. Inclined CFRP U wraps at ends

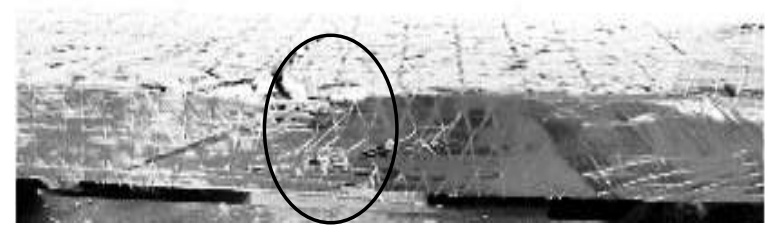

Figure 11. CFRP rupture at mid span

All the bonding techniques have proven their capability of delaying end-debonding failure and subsequent enhancement in ultimate failure loads with strength increments ranging from $60.68 \%$ to $213.46 \%$ and $14.64 \%$ to $31.21 \%$ in comparison of non-strengthened control test specimens and CFRP strengthened control test specimens respectively as illustrated in Fig. 12. The highest strength gain of $213.46 \%$ with regards to non-strengthened control test specimens corresponds to vertical CFRP U wraps whereas inclined CFRP U wraps achieved the highest strength increment of $31.21 \%$ compared to CFRP strengthened control specimens.

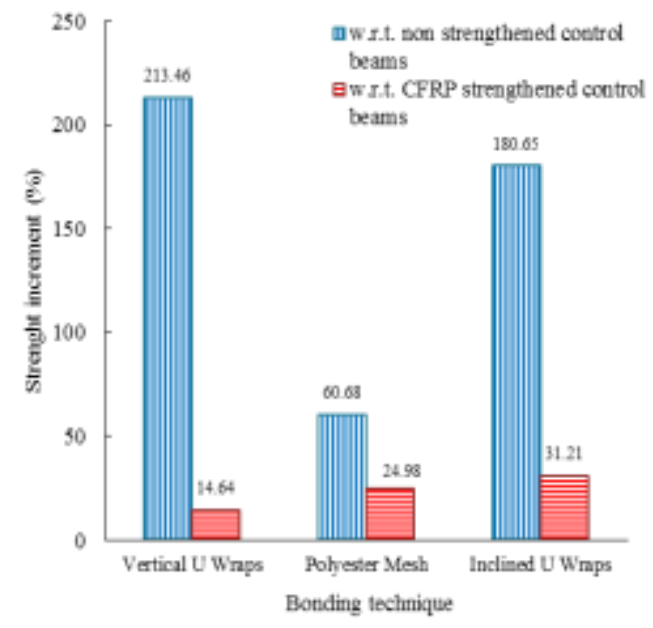

Figure 12. Comparison of strength gains from different bonding techniques

\section{Finite element modeling of CFRP anchors}

A three dimensional numerical model was developed using commercially available finite element package; ANSYS 12.0 performing a nonlinear static analysis. The main objective was to examine the efficiency of CFRP anchors to delay CFRP end-debonding failure and thereby upturn the ultimate failure loads CFRP/concrete composites. Concrete was represented by Solid 65 [11, 12], whereas steel reinforcements and anchor heads were modeled using Link 8 spar [11] element type. CFRP sheet and anchor heads were characterized by the element Shell 41 [11]. Concrete was meshed as hex diagonals of $25 \mathrm{~mm} \dot{\mathrm{x}} 25 \mathrm{~mm}$ and the meshed concrete beam is shown in Fig. 13. The Poisson's ratio of grade 30 concrete was assumed as 0.2 [13]. The elastic modulus of $27156.95 \mathrm{~N} / \mathrm{mm}^{2}$, uniaxial compressive strength of $29.5 \mathrm{~N} / \mathrm{mm}^{2}$ and uniaxial tensile strength of $4.43 \mathrm{~N} / \mathrm{mm}^{2}$ were obtained using the numerical expressions derived by Desayi and Krishnan [14] together with the measured average compressive strength of 33.6 $\mathrm{N} / \mathrm{mm}^{2}$ experimentally. Open and closed shear transfer 
coefficients were assumed as 0.5 and 0.9 [13] respectively. The material behaviour of steel was characterized as isotropic elastic - perfectly plastic material with bilinear Von Mises plasticity failure criterion. For both mild steel and galvanized iron materials, the material properties consisted of a Young's modulus of $200,000 \mathrm{~N} / \mathrm{mm}^{2}$, a Tangent modulus of 20,000 $\mathrm{N} / \mathrm{mm}^{2}$ and a Poisson's ratio of 0.3 were assumed [15]. The yield strengths of $490 \mathrm{~N} / \mathrm{mm}^{2}$ and $280 \mathrm{~N} / \mathrm{mm}^{2}$ were used for mild steel and galvanized iron materials respectively which were obtained via laboratory experiments. The CFRP sheet was modeled assuming linear orthotropic material behavior with nonlinear Hill plasticity failure criterion.

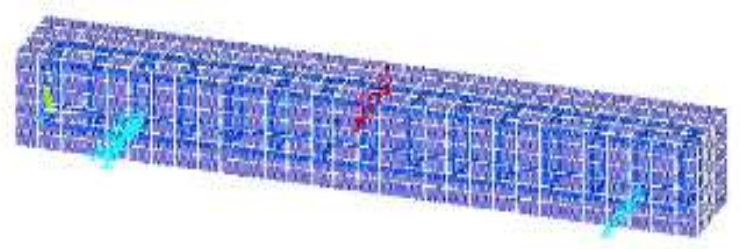

Figure 13. Meshed concrete beam

A three dimensional finite element model with $100 \mathrm{~mm} \times$ $150 \mathrm{~mm} \times 750 \mathrm{~mm}$ dimensions was modeled providing $25 \mathrm{~mm}$ cover and $50 \mathrm{~mm}$ spacing for shear links similar to the experiments. The CFRP/concrete composite beam was simply supported and the supports were located $100 \mathrm{~mm}$ inside from two edges. The developed nonlinear finite element model was validated with a considerable higher accuracy with merely $1.43 \%$ difference between numerical and experimental failure loads correspond to CFRP strengthened control test specimens. After the validation of the numerical model, three types of CFRP anchors which comprised Fan and Dowel anchor, pi-shaped anchor and tri-leg anchor were characterized as shown in Fig. 14, Fig.15 and Fig. 16 respectively.

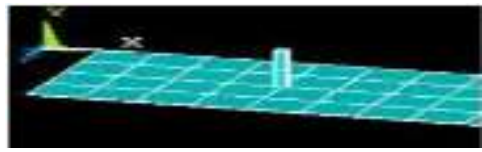

Figure 14. Numerical model of Fan and Dowel anchor
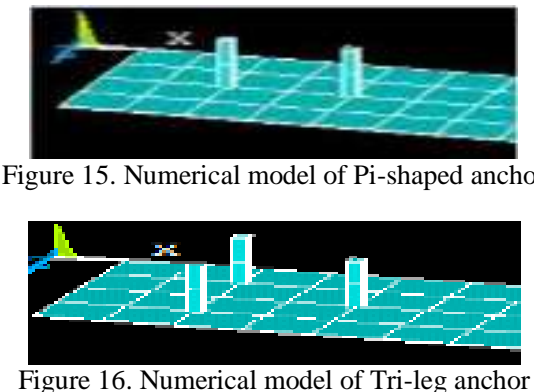

The geometry of newly proposed tri-leg anchor is illustrated in Fig. 17. The numerical analysis of CFRP/concrete composites with the three types of anchors were carried out based on the deflection study, failure load study, fibre strain study and crack pattern study. The obtained crack patterns are presented in Fig. 18.
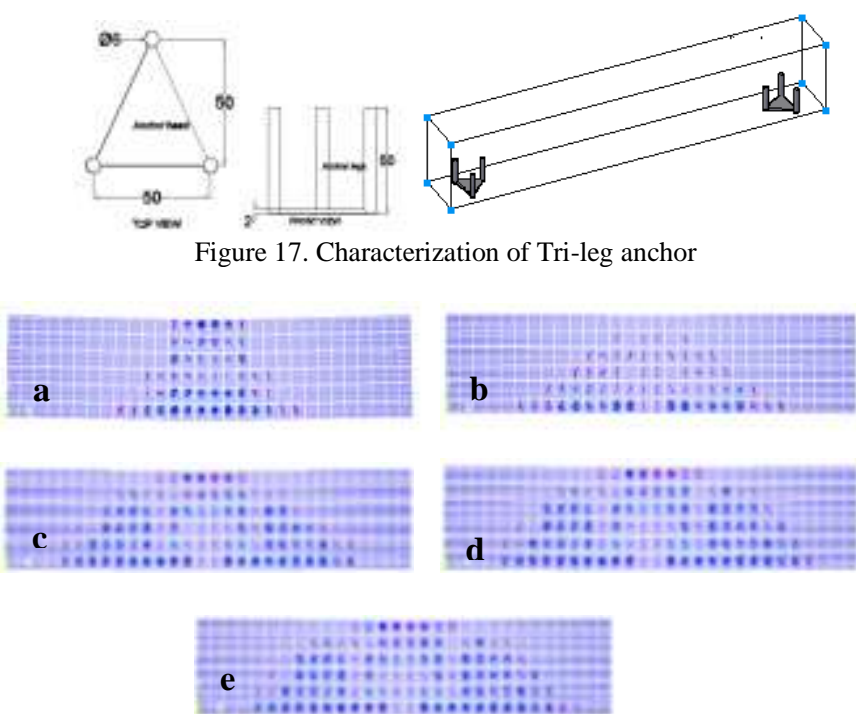

Figure 18. Crack patterns of a) Non-strengthened control beam b) CFRP strengthened control beam c) With Fan and Dowel anchor d) With Pi-shaped anchor e) With Tri-leg anchor

Non strengthened concrete beam showed flexural cracks at mid span as similar to which was observed in experiments. Cracks in CFRP strengthened concrete beams are further extended towards the ends of CFRP sheets. A comparable behavior was observed in experiments since the initiation of cracks occurred at ends of the laminate. Similarly, Fig. 18(c), (d) and (e) show almost similar behaviour as experiments.

All three types of anchors enhanced the failure loads of CFRP/concrete composites effectively reaching more than $211 \%$ strength increments in comparison of non-strengthened concrete test specimens. The newly proposed "Tri-leg anchor" has achieved the highest strength increment of $218.84 \%$ compared to non-strengthened control composite beams over other two types of anchors as shown in Fig. 19. The corresponding strength gains of Pi-shaped and Fan and Dowel anchors were $214.98 \%$ and $211.17 \%$ which comply with the previous research findings. Strength gains ranging from $18.6 \%$ to $68.7 \%$ have been reported for anchored CFRP/concrete composites compared to CFRP strengthened beams [16, 17].

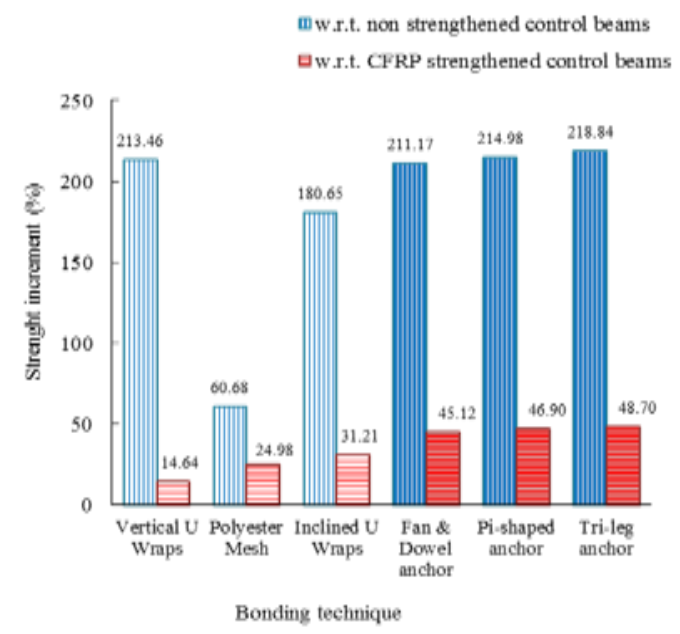

Figure 19. Comparison of strength gains from different bonding techniques including CFRP anchors 


\section{v. Conclusions and recommendations}

Premature debonding failure have been identified as the most critical failure mode of $\mathrm{CFRP/concrete} \mathrm{composites.}$ Many research studies have been focused on investigating techniques to delay debonding of CFRP sheet from the interface. A careful detailing at the CFRP sheet ends can significantly reduce the bond stress concentration which typically occurs at or near the strip ends. In the current study, four such different techniques have been proposed. The use of vertical and inclined $\mathrm{U}$ wraps at ends is a promising technique to ensure continuous transfer of force along the CFRP sheet. Moreover, the presence of a polyester mesh at bond line has proven to be a cost effective method which commendably distribute the interfacial stresses well. Three types of anchor systems were also investigated to enhance the performance of CFRP/concrete composites. Fan and Dowel anchor and Pishaped anchor have already been studied and the predicted results in the present study are almost comparable with the previous research findings. In the current study, a new anchor type has been proposed by authors which exhibited prominent strength increments over all other presented bonding techniques including two types of anchors. The effectiveness of anchoring systems with respect to non-strengthened control beam is listed below.

Tri-leg anchor ( $218.84 \%)>$ PI-shaped anchor $(214.98 \%)>$ vertical U wraps $(213.46 \%)>$ Fan and Dowel anchor $(211.17 \%)$.

Further research studies should be focused on preparation methods, geometry and configuration of Tri-leg anchor improving its competence.

\section{Acknowledgment}

This paper presents a summary of undergraduate research projects carried out at the Department of Civil Engineering, University of Moratuwa, Sri lanka. The authors would like to convey their appreciation to the research students H.R.D Premarathne, S. Srisangeerthanan, D.M.N. Wijerathna and U. Thanasree. The technical staff of Structural Testing Laboratory and Building Materials Laboratory at University of Moratuwa is also greatly acknowledged.

\section{References}

[1] A. Nezamian, S. Setunge, W. Lokuge et al, "Review of strengthening techniques using externally bonded fibre reinforced polymer composites", Report 2002-005-C-01, Australia: Construction Innovation, pp. 1-48, 2002.

[2] M.R. Aram, C. Czaderski and M. Motavalli, "Debonding failure modes of flexural FRP-strengthened RC beams", Composites: Part B, vol 39, pp. 826-841, 2008.

[3] J. Yao and J.G. Teng, "Plate end debonding in FRP-plated RC beamsI: Experiments", Engineering Structures, vol. 29, pp. 2457-2471, 2007.

[4] H. Pham, R. Al-Mahaidi, "Assessment of available prediction models for the strength of FRP retrofitted RC beams". Composite Structures, vol. 66, pp. 601-610, 2004.

[5] G.J Xiong, X. Jiang, J.W. Liu, and L. Chen, "A way for preventing tension delamination of concrete coverin midspan of FRP strengthened

beams", Construction and Building Materials, Elsevier Ltd, vol. 21, pp.402-408, 2007.

[6] Z. M. Wu, C.H. Hu, Y. F Wu and J.J. Zheng, "Application of improved hybrid bonded FRP technique to FRP debonding prevention", Construction and Building Materials, Elsevier Ltd, vol. 25, pp. 28982905, 2011.

[7] D. Mostofinejad and A. Moghaddas, "Bond efficiency of EBR and EBROG methods in different flexural failure mechanisms of FRP strengthened RC beams", Construction and Building Materials, Elsevier Ltd, Vol. 54, pp. 605-614, 2014.

[8] G. Spadea, R.N. Swamy and F. Bencardino "Strength and ductility of RC beams repaired with bonded CFRP laminates" Journal of Bridge Engineering, ASCE, vol 6, no 5, pp. 349-355, 2011.

[9] S.V. Grelle and L.H. Sneed, "Review of anchorage systems for externally bonded FRP laminaates", International Journal of Concrete Strucutres and Materials. vol. 7, no.1, pp. 17-33, 2013.

[10] MBrace CF 640/2600, Manufacturer specifications, BASF Chemical Company, 2013.

[11] M.C. Sundarraja and G. Ganesh Prabhu, "Finite element modelling of CFRP jacketed CFST members under flexural loading", Thin-Walled Structures, vol. 49, pp. 1483-1491, 2011.

[12] S.H. Alsayed, T.H. Almusallam, S.M. Ibrahim, N.M. Al-Hazmi, Y.A. Al-Salloum and H. Abbas, "Experimental and numerical investigation for compression response of CFRP strengthened shape modified walllike RC column", Construction and Building Materials, vol. 63, pp. 7280, 2014

[13] M. Y. H. Bangash, Concrete and Concrete Structures: Numerical Modelling and Applications, England:Elsevier Science Publishers, 1989.

[14] P. Desasyi and S.Krishnan, "Equation for the Stress-Strain Curve of Concrete", Journal of the American Concrete Institute, pp. 345-350, March 1964.

[15] J.Gere and S. Timoshenko, Mecahnics of Materials: Boston, Massachusettes: PWS Publishing Company, 1997.

[16] S.T. Smith, "FRP anchors: recent advances in research and understanding", Proceedings of $2^{\text {nd }}$ International Conference of FRP in Structures (APFIS), Seoul, Korea, 9-11 December, 2009.

[17] A. Ali, J. Abdalla, R. Hawileh and K. Galal, "CFRP mechanical anchorage for externally strengthened RC beams under flexure", Eighth International Conference on Material Sciences (CSM8-ISM5), Physics Procedia Vol.55 pp.10-16, 2014.

About Authors:
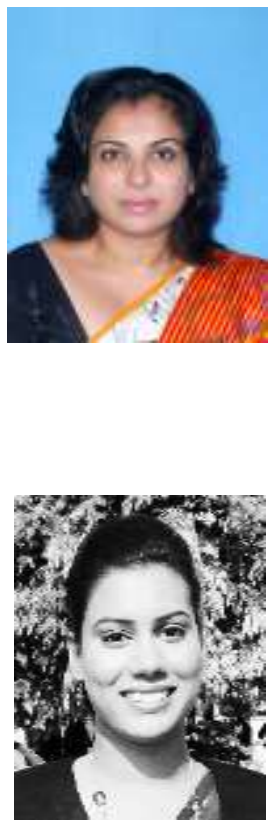

J C PH Gamage is a senior lecturer in the Department of Civil Engineering, University of Moratuwa, Sri Lanka. She obtained her bachelor degree from University of Moratuwa, MEngSc and $\mathrm{PhD}$ degrees from Monash University, Australia. She is a Chartered Engineer and has been involved in several consultancy projects especially in retrofitting structures. Her research interest includes retrofitting of structures, non linear finite element modelling, thin shell structures and inovative materials.

M.R.E.F Ariyachandra obtained her Bachelor's Degree from the University of Moratuwa, Sri Lanka in 2014. Currently, she is reading for her Master's Degree at the department of Civil Engineering, University of Moratuwa. Her research study is focused on durability performance of CFRP/concrete composites. 